\title{
Reflexionando sobre la práctica clínica: La autonomía de los pacientes adictos
}

Addiction and Autonomy

Yamila De Vas*

\begin{abstract}
Resumen
Se presenta el caso de un paciente alcohólico que en el escenario de una consulta por control de salud plantea abiertamente su negativa a cambiar el hábito ante la sugerencia del médico. Se discuten luego aspectos implicados en dicha entrevista, como ser: la negación, como principal mecanismo de defensa del adicto; la preservación o no de la autonomía del paciente; y la necesidad o no de intervención médica sobre la adicción.
\end{abstract}

\section{Abstract}

Upon a case presentation of an alcoholic patient who rejects medical advise during a regular medical visit, the authors discuss about denial as a defensive mechanism, whether patient's autonomy is preserved or not, and the appropriateness of medical intervention for the patient's addiction in this scenario.

Palabras clave: alcoholismo, adicción, negacion, autonomia, intervenciones. Key words: alcoholism, addiction, denial, autonomy, interventions.

De Vas Y, Pace R. La autonomía de los pacientes adictos. Evid Act Pract Ambul. Vol 13(3):96-98 Jul-Sept 2010

\section{Presentación del caso}

El caso es el de un paciente de 55 años con antecedentes de hígado graso, insuficiencia cardiaca, hipoandrogenismo y osteoporosis, muchas de estas enfermedades producto de su adicción al alcohol, que consulta a su médico de cabecera, quien se encuentra además acompañado por quien describe la situación.

Durante la consulta médica, el médico de cabecera hace hincapié en lo importante que sería para la salud del paciente el dejar de tomar alcohol, a lo que el paciente responde en forma tranquila y convincente que no tiene ningún interés de dejar el hábito ya que "...sin los placeres de la vida, la vida no tiene sentido...", que "...le gusta tomar..." y que él "...decide de que morir...".

Ante estas declaraciones reflexionamos junto al médico de cabecera sobre la posibilidad de que esta forma de pensar del paciente sea real o constituya una negación; si habría que aceptarla, o si por lo contrario, el médico debe y puede hacer una intervención para procurar que su paciente cambie de opinión.

\section{Preguntas que surgieron a partir del caso}

¿Se trata simplemente de una manifestación de negación de su enfermedad (adicción al alcohol)?

¿Cómo operar como médico respetando la opinión del paciente sin llegar a tratarlo como un enfermo carente de autonomía? ¿Puede un adicto tener autonomía, o justamente es lo que le falta al ser dominado por la bebida?

¿Qué intervenciones posibles existen para ser implementadas en dicho ámbito de atención?

\section{La negación y otros mecanismos implicados}

Sigmud Freud ${ }^{1}$ decía que la negación es una forma de protegerse de realidades desagradables con las que uno prefiere no lidiar y la clasificaba dentro de los mecanismos de defensa. En general suele negarse rechazando o ignorando los hechos empíricos o la evidencia incuestionable que acredita que lo negado existe y minimizando la importancia de aquello que está sucediendo. Sin embargo, el negador puede también aceptar los hechos empíricos y la evidencia incuestionable de que algo está sucediendo sin minimizar su importancia, pero adjudicándole la responsabilidad de su existencia, y su solución, a alguien más.

Por otro lado, varios autores ${ }^{2,3,4}$ sostienen que además de utilizar los mecanismos de negación y auto-engaño, el adicto hace uso frecuente de otros mecanismos defensivos entre los cuales se encuentran la proyección ${ }^{\S}$, el desplazamiento; la racionalización y la distorsión ${ }^{*}$; cuya finalidad sigue siendo el no reconocimiento de su realidad en relación con el consumo de la sustancia en cuestión, y el consecuente escape de la misma.

La ambivalencia es otra característica que se encuentra presente en el adicto ya que, de alguna manera, éste aspira a librarse de las consecuencias de su adicción; pero también desea, simultáneamente, seguir "disfrutando" de los efectos (estimulantes, sedantes, etc.) que la sustancia consumida le provoca. Muchos coinciden en que la solución de este conflicto resulta de vital importancia para que un adicto inicie un tratamiento efectivo.

La caracterización del adicto es algo que resulta muy conveniente, sobre todo cuando se desea aumentar la eficacia de cualquier intervención que vaya dirigida a su tratamiento.

\section{La autonomía}

La autonomía (del griego auto, "uno mismo", y nomos, "norma") es, en términos generales, la capacidad de tomar decisiones sin ayuda de otro, sin influencia de presiones internas y externas, sinónimo de independencia y libertad ${ }^{5}$. Como vemos, esta definición se contrapone al concepto de dependencia alcohólica.

El principio de autonomía exige el respeto a la capacidad de decisión de las personas y el derecho a que se respete su voluntad, en aquellas cuestiones que se refieren a ellas mismas. Sin embargo el alcoholismo genera en forma directa o indirecta muchísimos problemas que afectan a terceros.

Mencionamos entre ellos el deterioro de las relaciones familiares, laborales y sociales; las lesiones no intencionales domésticas, laborales y de transito; y las conductas violentas. Por ejemplo, el consumo excesivo de alcohol es la tercera causa de muerte evitable en los Estados Unidos ${ }^{6}$, está relacionado con el $40 \%$ de las muertes producidas por lesiones de tránsito, con el 15 al $20 \%$ de las producidas en el ámbito de trabajo y con el $50 \%$ de los homicidios; razones por las cuales el equipo de salud no debería dejar de intervenir sobre el paciente durante cada consulta con el objetivo de indagar y prevenir estas situaciones.

\section{Las intervenciones}

Dentro de las intervenciones que puede realizar el médico de cabecera existe un modelo de seis pasos ${ }^{7}$, el cual resumimos a continuación: 
1) Identificación de los pacientes que beben más allá de los límites consensuados hoy como normales. El calculo del alcohol consumido puede cuantificarse por unidades (UBE: Unidad de Bebida Estándar, $1 \mathrm{U}=10 \mathrm{~g}$ de alcohol). Un vaso de vino de $100 \mathrm{~mL}, 200 \mathrm{~mL}$ de cerveza y $25 \mathrm{~mL}$ de whisky equivalen 1 UBE. Se habla de bebedor de riesgo cuando el consumo de alcohol es mayor a 28 UBE semanales en hombres y 17 UBE en mujeres, o bien a 5 UBE durante 24 horas por lo menos una vez por mes, o cualquier grado de consumo en pacientes con antecedentes familiares de alcoholismo ${ }^{2}$.

2) Diagnóstico preciso del nivel de adicción en que se encuentra el individuo. Para detectar abuso de alcohol puede utilizarse el cuestionario $\mathrm{CAGE}^{9}$ (ver tabla 1) mientras que el diagnóstico de síndrome de dependencia alcohólica puede establecerse de acuerdo a los criterios del DSMIVV ${ }^{10}$ (ver tabla 2 ).

3) Educación del paciente y su familia sobre lo que se ha identificado en los pasos anteriores, discutiendo en forma particular con cada paciente los riesgos y los beneficios de beber alcohol; y reflexionando sobre que es lo que consigue con el alcohol que no pude conseguir sobrio. Durante este paso es importante evitar formular juicios de valor y estigmatizar al paciente con el término "alcohólico", siendo útil intentar vincular al alcohol con algún otro problema de salud que el paciente tenga "registrado" e insistir en que dejando de tomar alcohol mejorará ese problema.

4) Llegar a un mutuo acuerdo junto al paciente respecto del problema, reconociendo los riesgos vinculados con la ingesta de alcohol. Si el desacuerdo entre el equipo de salud y el paciente es muy grande, se recomienda revalorar la situación y volver a intervenir educativamente antes de elegir un plan de manejo definitivo.

5) Negociar con el paciente y la familia un plan definitivo para abordar su adicción basado en el mutuo acuerdo.

6) Acordar con el paciente un plan de seguimiento para observar y monitorear la efectividad del plan de tratamiento estando preparados ante una eventual recaída.

A diferencia de los tomadores pesados y los malos tomadores, con quienes se puede negociar moderar el consumo de alcohol, cuando se trate de individuos abusadores y dependientes, el objetivo del equipo tratante debe ser que adhieran a un programa de tratamiento que incluya la abstinencia como principio fundamental ${ }^{7}$.

Los médicos no deberían ir mucho más rápido que los pacientes ni intentar "curar" las adicciones durante un par de consultas. Se recomienda ${ }^{7}$ dar los pasos "uno tras otro", respetando el momento y las fases por las que atraviesa cada paciente. Como en todas las adicciones el pronóstico es malo y sólo un porcentaje muy bajo podrá ser ayudado por el sistema médico para lograr la recuperación.

El principal desafío del médico será realizar el diagnostico que muchas veces no es tan sencillo y estar capacitado para evaluar correctamente al paciente y ofrecerle ayuda, conociendo las implicancias familiares para poder trabajar con ellas, y asumiendo que la enfermedad (o por lo menos su repercusión) abarca toda la familia.
Tabla 1. Cuestionario CAGE

\begin{tabular}{l} 
1) Le ha molestado alguna vez la gente criticándolo por su forma de beber? \\
\hline 2) Ha tenido alguna vez la impresión de que debería beber menos? \\
3) Se ha sentido en alguna ocasión mal o culpable por su costumbre de beber? \\
4) En alguna ocasión lo primero que ha hecho en la mañana es beber para calmar \\
nervios o librarse de alguna resaca?
\end{tabular}

Dos o más respuestas afirmativas indican dependencia al alcohol ${ }^{10}$.

Tabla 2. Criterios diagnósticos del DSMIV para dependencia alcohólica.

\begin{tabular}{l|l}
\hline Tolerancia & $\begin{array}{l}\text { Necesidad de consumir cantidades crecientes de alcohol } \\
\text { para lograr el efecto deseado o disminución del efecto tras } \\
\text { consumir la misma cantidad. }\end{array}$ \\
\hline Deprivación & $\begin{array}{l}\text { Ingesta de alcohol o sustancia relacionada para aliviar o } \\
\text { evitar síntomas de deprivacion. }\end{array}$ \\
\hline Alteración de control & $\begin{array}{l}\text { Deseo persistente o bien uno o más intentos de reducir 0 } \\
\text { controlar el consumo de alcohol: ingestas de mayores } \\
\text { cantidades o durante periodos de tiempo más prolongados } \\
\text { que los planteados. }\end{array}$ \\
\hline Descuido de & $\begin{array}{l}\text { Abandono o disminución importante de las actividades } \\
\text { sociales, laborales o recreativas por causa de la bebida. }\end{array}$ \\
\hline actividades & $\begin{array}{l}\text { Mucho tiempo utilizado en actividades necesarias para } \\
\text { obtener alcohol o recuperarse. }\end{array}$ \\
\hline alcohol & Consumo de alcohol a \\
pesar de problemas & $\begin{array}{l}\text { Consumo continuado a pesar de los persistentes problemas } \\
\text { físicos y psicológicos que pueden deberse a ello. }\end{array}$ \\
que ocasiona & Peno de
\end{tabular}

Se considera que hay un problema si se cumplen 3 o más criterios durante el último año ${ }^{10}$.

\section{¿Qué opina un Comité de Bioética Asistencial?}

Luego de encontrar en la bibliografía consultada diferentes enfoques sobre el principio de autonomía hemos decidido llevar el caso al Comité de Bioética Asistencial del Hospital Italiano de Buenos Aires para conocer la opinión al respecto de este grupo. Tras presentar el caso, distintas y divididas fueron las opiniones en cuanto a la presencia o no de autonomía de este paciente. Algunos integrantes del Comité defendían por sobre todas las cosas el principio de autonomía de las personas y creían, sin dudar, en el discurso de éste paciente; por el contrario, otros hacían hincapié en la falta de autonomía mientras se encontraba alcoholizado y anclándose en el mecanismo de negación, ponían en duda la preservación de la misma estando el paciente sobrio.

Se llegó a la conclusión de que como en muchos aspectos de la vida y de las relaciones humanas, las calificaciones no pueden ser absolutas sino que tienen diferentes gradaciones. La autonomía no escapa a este concepto, por lo que cada caso debe ser evaluado como único, debiendo siempre el equipo de salud intentar dejar fuera del análisis su apreciación personal

SLa proyección es un mecanismo de defensa a través del cual el individuo se enfrenta a conflictos emocionales y amenazas de origen interno o externo atribuyendo incorrectamente a los demás sentimientos, impulsos o pensamientos propios, que le resultan inaceptables o que le generan negación. Consiste en proyectar cualidades, deseos o sentimientos que producen ansiedad fuera de sí mismo, dirigiéndolos hacia algo o alguien a quien se los atribuyen totalmente. Un ejemplo de ello es cuando se justifica la tardanza diciendo que se ha llegado tarde porque los demás también son impuntuales, cuando en realidad puede que esto sea totalmente incierto

'En psicología, desplazamiento es un mecanismo de defensa inconsciente en que la mente redirige algunas emociones de un objeto y/o representación psíquica (por ejemplo, en los sueños) que se percibe como peligroso o inaceptable a uno aceptable. Por ejemplo: un empleado puede desquitarse con su esposa cuando regresa del trabajo después de haber sido humillado por su jefe. El desplazamiento puede accionarse en cadena. Siguiendo el ejemplo de arriba, la esposa golpeada puede golpear al hijo, quien a su vez puede golpear al perro; cada sujeto justificando su conducta a través de racionalizaciones. El mecanismo inconsciente del desplazamiento se usa para eludir la angustia.

${ }^{*}$ La racionalización es un mecanismo de defensa que consiste en justificar las acciones (generalmente las del propio sujeto) de tal manera que eviten la censura. Se tiende a dar con ello una "explicación lógica" a los sentimientos, pensamientos o conductas que de otro modo provocarían ansiedad o sentimientos de inferioridad o de culpa; de este modo una racionalización o un transformar en pseudorazonable algo que puede facilitar actitudes negativas ya sean para el propio sujeto o para su prójimo. Un ejemplo es el caso de una persona que emplea humor cáustico en una relación interpersonal, y lo justifica interpretándolo como "juego" o "diversión", y no como una crítica o actitud agresiva. Es importante hacer notar que, para ser considerada racionalización, el sujeto debe creer en la solidez de su argumento, no empleándolo como simple excusa o engaño consciente. 
sobre el tema y reconocer el derecho del paciente a pensar y sentir distinto.

La Bioética estudia la conducta humana en el ámbito de las ciencias de la vida y de la salud, analizada a la luz de los valores y la moral. Se basa en cuatro principios: justicia, autonomía, beneficencia y no maleficencia. En la antigüedad, la formula "favorecer o no perjudicar" significaba la unión del principio de beneficencia y no maleficencia; la separación de ambos apareció con el concepto de autonomía. En aquel tiempo, la beneficencia era tomada como lo que hoy llamamos paternalismo, que consiste en una beneficencia sin tener en cuenta las preferencias del paciente. Así, los principios eran interpretados desde la óptica del médico exclusivamente y nunca desde la del enfermo, quien solo acataba las indicaciones del médico como único conocedor de la verdad y de lo que era mejor para el paciente. Las raíces de este accionar médico podrían encontrarse en el Juramento Hipocrático. Con la formulación de los derechos humanos aparece en escena la autonomía y comienza a cuestionarse todas las posturas paternalistas.

La ética moderna pone a la autonomía como principio por excelencia de todo ser humano y la falta de esta conlleva irremediablemente a la perdida de toda dignidad. Tal es así que en noviembre de 2009 se promulgó en Argentina la Ley 26.529 sobre los derechos del paciente, explicitándose el derecho a la autonomía de la voluntad de todo individuo.

El concepto de autonomía total debe ser considerado solo como un ideal ya que hay factores que continuamente influyen en la capacidad individual de tomar decisiones autónomas ${ }^{11,13}$. Uno de estos factores puede ser la presencia de enfermedades o adicciones que hagan perder a la persona la capacidad de controlar sus acciones.

En principio, la adicción no debería ser considerada por si misma una fuerza destructora de la autonomía de la persona, a menos que la persona desee fervientemente no ser adicta y no lo logre. Otro factor a considerar es la forma de razonar de cada individuo, ya que para que sus decisiones y acciones sean autónomas deben tener algún tipo de fundamento en el medio cultural que vive; si solo se trata de su apreciación personal, su autonomía estaría restringida. Un tercer factor es la estabilidad en el tiempo de nuestras decisiones, ya que ser autónomo significa actuar en base a las creencias del momento, aunque en un pasado o en un futuro se hubiera pensado distinto. Como último factor, aparece el que mas seriamente implica al equipo de salud tratante: brindar al paciente información completa y verdadera, para que pueda tomar una decisión en la forma más autónoma posible.

\section{Conclusión}

Luego de analizar los factores que alteran la práctica de la autonomía de cada individuo, podemos observar que la autonomía perfecta o completa es prácticamente un pensamiento ideal, probablemente inalcanzable y que seguramente no haya una respuesta única que responda si la autonomía se encuentra preservada o no en los adictos en general, sino que como en cualquier problema ético, los casos (al igual que las personas) deben ser analizados individualmente.

Recibido el 03/07/2010 y aceptado el 01/08/2010

\section{Referencias}

1. Sigmund Freíd. La Negación, (1925). Obras Completas. Biblioteca Nueva. Madrid, 1981

1. Alvarado S. Perfil psicológico de la persona adicta. Madrid: Editorial Pirámide; 1996.

3. Mansilla JC. El problema de los valores y la ética en la rehabilitación del adicto. Citado por Zaldívar Pérez D, Caracterización psicológica del adicto, disponible en: http://www.sld.cu/saludvida/adicciones/temas.php?idv=5763 (último acceso 20/08/10).

4. Zaldívar D. La intervención psicológica. Michoacán, México: Editorial IMCDE; 2001.

5. Diccionario Real Academia Española. Disponible en URL: http://www.rae.es/rae.html (último acceso 20/08/10)

6. Gold M, y col. Screening for and diagnosis of patients with alcohol problems. Epidemiology and Risk Factors. In: UpToDate, Basow, DS (Ed), UpToDate, Waltham, MA, 2007. 7. Limanski R. Alcoholismo. En: Medicina Familiar y Práctica ambulatoria. Editores. Rubinstein A, Terrasa S y col. 2 da edición, Buenos Aires, Médica. Panamericana, 2007.

8. Grupo de Educación Sanitaria y Promoción de la Salud del PAPPS. Sociedad Española de medicina Familiar Y Comunitaria. (semFYC) Barcelona 2005. Disponible en URL http://www.semfyc.es (último acceso 20/08/10).

9. Ewing J,y col. Screening for Alcoholism Using CAGE. JAMA. 1998;280(22):1904-1905.

10. First M, y col. DSM IV- TR: Manual diagnostico Diferencial. Barcelona, 2002.

11. Pace R, Caffaro Hernandez N. Iniciación a la Bioética. Capitulo Autonomía. Delhospitalediciones. 2008. 